\title{
Radiation Therapy Improves Local Control in Juvenile Nasopharyngeal Angiofibroma following Disease Progression after Embolization and Surgical Resection: A Case Report
}

\author{
Zane Blank Richard Sleightholm Beth Neilsen Michael Baine Chi Lin \\ Department of Radiation Oncology, University of Nebraska Medical Center, Omaha, NE, \\ USA
}

\section{Keywords}

Juvenile nasopharyngeal angiofibroma · Otorhinolaryngology · Pediatrics · Radiation therapy · Surgery

\begin{abstract}
Juvenile nasopharyngeal angiofibroma (JNA) is a relatively uncommon, benign neoplasm of the nasopharynx that can be very difficult to diagnose early due to inconspicuous and seemingly harmless presenting symptoms. Early diagnosis and treatment of JNA are essential for a good prognosis. JNA typically responds well to radiation therapy (RT), but when it does not, the most appropriate next course of action has not been readily defined due to the limited occurrence and experience with this neoplasm. Herein, we describe a JNA patient, who continued to progress after surgery and 36 Gy of adjuvant radiation, but after an additional 14.4 $\mathrm{Gy}$, he has remained in remission for over 2 years. An 11-year-old boy who presented with JNA underwent treatment with embolization and surgical resection. Unfortunately, the tumor progressed within 2 months of surgical intervention and he required RT for adequate local control. While undergoing RT, he again demonstrated signs of progression; so his radiation regimen was increased from 3,600 cGy in 20 fractions to 5,040 cGy in 28 fractions. Since completing RT, the tumor has continued to decrease in size, and the patient is stable and has been without signs of disease progression for over 24 months now. Thus, escalating the radiation regimen to 5,040 cGy may improve local control in rapidly progressive JNA.
\end{abstract}

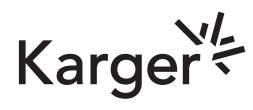




\section{Case Reports in Oncology}

\begin{tabular}{l|l}
\hline Case Rep Oncol 2021;14:739-745 \\
\hline DOI: 10.1159/000512061 & $\begin{array}{l}\text { @ 2021 The Author(s). Published by S. Karger AG, Basel } \\
\text { www.karger.com/cro }\end{array}$ \\
\hline
\end{tabular}

Blank et al.: Radiation Therapy Improves Control in Juvenile Nasopharyngeal Angiofibroma following Surgery

\section{Introduction}

Juvenile nasopharyngeal angiofibroma (JNA) is a relatively uncommon, benign neoplasm of the nasopharynx, with an incidence of 1 case per 150,000 individuals. Although it can occur at any age, young adults and adolescents between the ages of 14 and 25 are primarily affected, and there is a significantly distinct male predominance [1]. The sex selectivity and young population of JNA suggest that the origin of tumor development is hormone dependent. Several investigators have studied various receptors, including androgen, progesterone, and estrogen; however, there have been discrepancies in the results [1]. Although benign, JNA is a very locally aggressive tumor and commonly invades the surrounding tissues and bone. Previously, the Fisch classification described the severity of the disease based on the extent of local invasion [2]. Though there remains a lack of universal acceptance of staging, the most recent system, the Radkowski JNA Classification System (Table 1), emphasizes the importance of posterior extension and the degree of invasion into the skull base [3].

Classically, JNA presents as a progressive, unilateral nasal obstruction with or without epistaxis and rhinorrhea. It is typically painless. The rich vasculature and lack of encapsulation allow for aggressive local invasion of adjacent structures. Depending on the area of invasion, it can cause facial deformities, changes in vision, proptosis, and Eustachian tube dysfunction. Diagnoses are made by strong clinical suspicion, a complete history, nasal endoscopy, and various imaging modalities including CT, MRI, and arteriography which allow proper visualization of the tumor site and to establish the extension of the tumor into adjacent structures. Due to the characteristic findings of JNA on CT and angiography, in addition to the major bleeding risk, biopsy should be avoided in the initial evaluation.

Early diagnosis and treatment in JNA are essential for a good prognosis. This can be very difficult, however, given the seemingly harmless presenting signs and symptoms. Delayed diagnoses allow the tumor to advance and it becomes more challenging to treat. Recurrence rates are higher and correlate directly to the stage of the tumor [4, 5]. Given the highly vasculature nature of JNA, patients are typically treated with preoperative embolization followed by surgical resection. Embolization has proven to significantly reduce bleeding intraoperatively and the need for blood transfusions [6]. Surgical resection is the primary treatment modality. However, in later stages, there is dispute as to how to treat JNA. More extensive tumor invasion may necessitate a combination of treatment modalities, including surgical resection and postoperative radiation therapy (RT). In extracranial cases, surgical resection with or without embolization is recommended. In cases with residual disease, the tumor may

Table 1. Radkowski JNA Classification System stages

\begin{tabular}{|c|c|}
\hline Stage & Features \\
\hline IA & Limited to nose and/or nasopharyngeal vault \\
\hline IB & Extension into $\geq 1$ paranasal sinus \\
\hline \multirow[t]{2}{*}{ IIA } & Minimal extension into sphenopalatine foramen \\
\hline & Includes minimal part of medial pterygomaxillary fossa \\
\hline \multirow[t]{3}{*}{ IIB } & Full occupation of pterygomaxillary fossa with Holman-Miller sign \\
\hline & Lateral or anterior displacement of maxillary artery branches \\
\hline & May have superior extension with orbital bone erosion \\
\hline IIC & $\begin{array}{l}\text { Extension through pterygomaxillary fossa into cheek, temporal fossa, or posterior to } \\
\text { pterygoids }\end{array}$ \\
\hline IIIA & Skull base erosion with minimal intracranial extension \\
\hline IIIB & $\begin{array}{l}\text { Skull base erosion with extensive intracranial extension }+/- \text { cavernous sinus involvement } \\
\text { pterygomaxillary fossa }\end{array}$ \\
\hline
\end{tabular}


be observed unless symptoms develop for which RT should be implemented. However, in JNA cases that are intracranial, orbital, or involve pterygopalatine extension, RT to a dose of 30-50 Gy in 2-3 Gy fractions is recommended [7-10]. Local control of JNA with RT is approximately $80 \%$, but tumors may take up to 2 years to regress [10]. It is recommended that patients be followed with imaging every 6 months for at least 5 years post-RT to ensure the disease does not progress.

Here we present the case of an 11-year-old male who was diagnosed with Fisch stage IVb JNA. He underwent preoperative embolization followed by a subtotal surgical resection. Based on follow-up imaging and the concern for progression of the residual tumor, he subsequently underwent 3,600-cGy external beam RT to the nasopharynx. Due to the level of resistance after the initial doses of radiation, the dose was increased to a total of 5,040 cGy in 28 fractions. After 18 months post-RT, the patient is doing well, and his disease has remained in remission.

\section{Case Presentation}

At initial presentation, an 11-year-old male patient presented in the emergency room with a 1-month history of congestion, rhinorrhea, and snoring. On physical exam, he was found to have a high arching, floppy posterior soft palate and inflamed nasal turbinates. He also exhibited left eye visual field deficits, which the patient said had been present for 2 weeks. He described the deficit as starting as a small black dot that slowly progressed to complete blackness. The patient was referred for an immediate full dilated eye exam, where the optometrist ordered an MRI of the brain and orbits. In addition, ambulatory referral to ENT was placed for evaluation of his abnormal palate and differentiation of persistent allergic rhinitis and/or bacterial sinusitis. The emergency department discharged him with cetirizine and oxymetazoline for congestion and counseled him to return if symptoms continued or acutely worsened.

Four days later, the patient got an orbit and sinus MRI that showed a large multi-spatial nasopharyngeal and nasal cavity mass involving the left sphenopalatine foramen that extended anteriorly into the nasal cavity along the turbinates reaching the left nasal passage, posteriorly to invade and replace the bony sella, laterally through the pterygomaxillary fissure, and caudally anterior to the adenoids with obliteration of the nasopharyngeal airway (Fig. 1). The mass measured approximately $7 \times 7 \times 7 \mathrm{~cm}$. CT of the facial bones also showed the large multi-spatial nasopharyngeal and nasal cavity mass with associated destructive and expansile bony changes. Due to these findings, he was subsequently admitted for inpatient evaluation. He underwent bilateral nasal endoscopy with biopsy of the left nasal mass. The left nasal cavity was noted to be involved with extensive mass extending under the inferior turbinate to the anterior aspect of the turbinate and along the length of the nasal cavity. The right nasal cavity was mostly obstructed by severe septal deviation and mass effect from the left-sided tumor. Pathology demonstrated highly vascular benign-appearing mesenchymal proliferation, most likely representing capillary hemangioma. The differential diagnosis included solitary fibrous tumor and JNA. Further evaluation confirmed that the specimen had features consistent with JNA and was secondarily validated by an outside laboratory.

One week after initial presentation, the patient underwent neuroembolization. The operation was completed without complications. Two days later, imaging still demonstrated a large, heterogeneous hypervascular nasopharyngeal mass compatible with JNA now with interval embolization of multiple left-sided feeding arteries (Fig. 1). One week after embolization, the patient underwent a subtotal resection via bitemporal craniotomy with translabial and transnasal endoscopic and open-resection approach with split thickness bone graft and

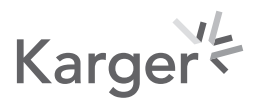



in Oncology

\begin{tabular}{l|l}
\hline DOI: 10.1159/000512061 & $\begin{array}{l}\text { O 2021 The Author(s). Published by S. Karger AG, Basel } \\
\text { www.karger.com/cro }\end{array}$ \\
\hline
\end{tabular}

Blank et al.: Radiation Therapy Improves Control in Juvenile Nasopharyngeal Angiofibroma following Surgery

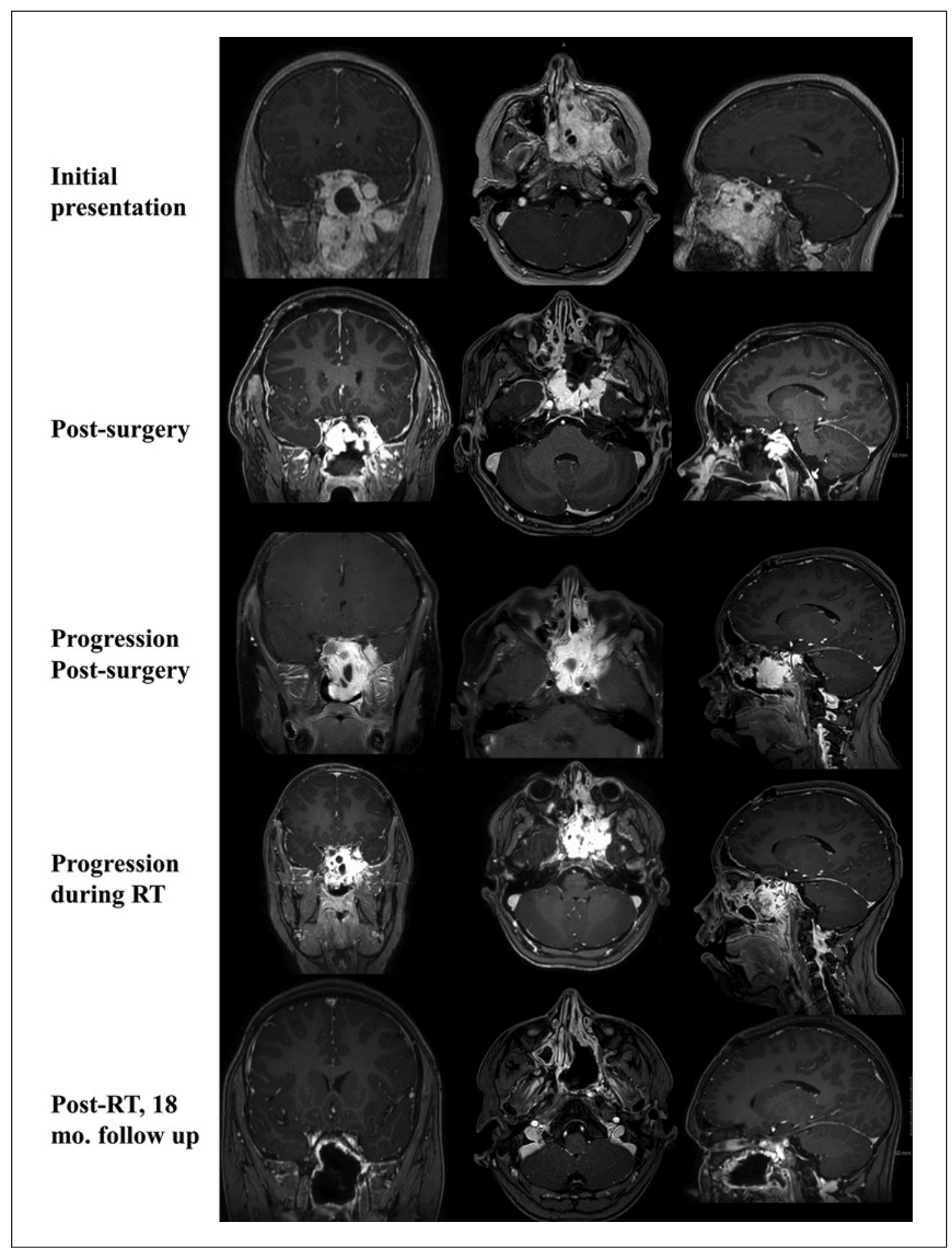

Fig. 1. Disease at initial presentation (first row), after surgery (second row), upon post-surgical progression (third row), during RT (fourth row), and at most recent follow-up 18 months postcompletion of extended RT treatment (fifth row). 
Blank et al.: Radiation Therapy Improves Control in Juvenile Nasopharyngeal Angiofibroma following Surgery

anterior cranial base repair. The pathology of the biopsy taken during surgery demonstrated features consistent with JNA. The following day, MRI of the brain and face demonstrated postoperative changes, and subtotal resection of the large nasopharyngeal mass with residual measuring $2.3 \times 4.4 \times 2.9 \mathrm{~cm}$ decreased from the previously measured $7.5 \times 5.7 \times 7.6 \mathrm{~cm}$. Imaging demonstrated the cavernous portion and a small portion of the sphenoid part of the tumor was not resected.

Approximately 1 month after resection, follow-up imaging demonstrated postoperative changes to the facial bones consistent with prior partial resection of the anterior aspect of the nasopharyngeal mass with a residual $3.9 \times 2.8 \times 2.9 \mathrm{~cm}$ heterogeneous enhancing mass with extensive unchanged osseous erosion predominantly involving the sphenoid body. Given contrast timing and venous enhancement of the cavernous sinuses, involvement of the left cavernous sinus could not be definitively excluded. Follow-up endoscopic exam 2 months after resection revealed that the tumor remnant was starting to enlarge. Therefore, the patient was referred to radiation oncology to discuss treatment options. Subsequently, it was recommended the patient undergo external beam RT to the residual tumor to a dose of 3,600 cGy in 20 fractions for improved local control. The patient started his RT approximately 2 months after completing surgical resection and 3 months from initial diagnosis.

During RT, close monitoring revealed improvement in visual symptoms as the patient reported that his left eye vision had improved, noting he now saw gray instead of complete blackness. Interval MRI demonstrated likely progression of disease (Fig. 1); so his prescription was increased to 5,040 cGy in 28 fractions. He completed the entirety of 5,040 cGy over 5 and a half weeks without complications or side effects.

Two months after RT completion, a follow-up brain MRI showed decreased residual JNA at the paranasal sinus region with signs of previous embolization, surgical, and radiation treatments. Discrete macrocystic foci had decreased in size since the most recent prior MRI. Close follow-up occurred every 3-4months, and MRI 6 months postcompletion of RT demonstrated a further decrease in tumor size of $1.7 \times 1.3 \times 1.6 \mathrm{~cm}$. At 10 months, the patient was doing well with no evidence of disease progression, and follow-up visits were lengthened to every 6 months. Repeat imaging at 18 months revealed the tumor had decreased further in size now measuring $1.7 \times 0.9 \times 1.1 \mathrm{~cm}$ (Fig. 1), and the patient has remained stable without signs of disease progression for over 24 months since the completion of his RT.

\section{Discussion/Conclusions}

Initial surgical intervention has been the conventional approach to JNA with RT reserved for the treatment of tumors that recur or are unresectable due to intracranial extension. Unfortunately, a significant portion of patients who undergo surgery will have tumor recurrence that requires additional treatment, and in these patients, postoperative radiation should be considered. RT may also be preferred in patients that are considered unresectable due to intracranial extension. For example, one case series described 3 cases of large JNAs with extensive intracranial extension that were primarily managed with external beam RT treated with 36.6, 40, and 50.4 Gy with good long-term outcomes [11]. While surgical intervention has been preferred for the primary treatment of these tumors if resectable, recent evidence has shown that RT is similarly efficacious as an initial intervention. In one retrospective study, the resolution of symptoms following neoadjuvant RT was 78.5\% (10/14) versus $76.5 \%$ in a comparable cohort who received upfront surgery [12]. In another retrospective study by Lee et al. [7], patients treated with primary RT (30-55 Gy) had a local control rate of $85 \%$ at 2 years, a similar rate to Cummings et al. [13] of $83 \%$ (35/42) at $>3$ years.

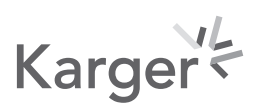


It should be emphasized that RT regimens for the treatment of JNA have been highly variable due to its relatively rare nature and lack of formalized studies evaluating dose efficacy. Retrospective studies have revealed improved control to over 90\% with 35-36 Gy versus 77\% with 30-32 Gy [8]. These dosing recommendations stem from retrospective studies of patients treated at UCLA between 1960 and 1985 that found isolated residual and symptomatic disease in 3 patients who received less than 36 Gy [12]. In another study, 4 out of 5 patients that received $32 \mathrm{~Gy}$ had recurrence within 2 years, while none of the 10 who received 34-36 Gy recurred [14]. Finally, in one retrospective review of 22 patients who received between 30 and 36 Gy (10 definitive RT and 12 postsurgical resection with evidence of recurrence), local control was $91 \%$. All of the failures occurred in patients who received 30 or $31.8 \mathrm{~Gy}$, while none of the 9 patients receiving at least 35 Gy developed recurrence further lending credence to RT regimens of $\geq 35$ Gy providing superior control [15]. For inoperable tumors or tumors that progress during RT, increasing the dose to $50 \mathrm{~Gy}$ is warranted, but no elective nodal RT is recommended $[10,14,16]$.

The benefits of RT should be weighed with the risk of long-term complications incluing growth retardation, panhypopituitarism, temporal lobe necrosis, cataracts, and radiation keratopathy with a complication rate of $15 \%$ in one study [7]. However, the rates of these long-term complications have decreased with the advent of improved technologies such as external beam RT, which more accurately deliver radiation to target tumors and minimize exposure to nearby critical structures. In a more recent study, the only side effects after 36-52 Gy RT seen in 13 JNA patients ( 2 initial therapy and 11 following surgery) were xerostomia and caries [16]. Furthermore, MRI should be used to help identify disease extension as well as for proper external beam RT contouring to further mitigate off-target effects. High-quality imaging and current technologies also allow for reduced expansion volumes with the CTV being GTV $+0.5 \mathrm{~cm}$ without exceeding anatomical boundary and PTV being expanded between 3 and $5 \mathrm{~mm}$ from the CTV, which is institutional dependent, and further contribute to improved outcomes and reduced side effects.

Herein, we present the case of an 11-year-old male with JNA that underwent embolization and subtotal surgical resection. Though JNAs are benign, they can be locally invasive and compress critical structures such as nerves and vessels, and the residual tumor in this patient unfortunately enlarged postsurgery, prompting treatment with RT. Initially 36 Gy in 20 fractions was planned based on previous studies suggesting a high level of control at that dose $[7,12]$. However, the patient demonstrated signs of continued progression while undergoing RT prompting re-evaluation and escalation to 50 Gy in 28 fractions. Thus far, this patient has remained symptom-free for over 2 years.

\section{Acknowledgements}

We appreciate the patient and his family for allowing the presentation of this case and helping the medical community in our understanding JNA.

\section{Statement of Ethics}

Written informed consent was obtained from the patient and his guardians for publication of this case report and any accompanying images. 


\section{Case Reports in Oncology}

\begin{tabular}{l|l}
\hline Case Rep Oncol 2021;14:739-745 \\
\hline DOI: 10.1159/000512061 & $\begin{array}{l}\text { @ 2021 The Author(s). Published by S. Karger AG, Basel } \\
\text { www.karger.com/cro }\end{array}$ \\
\hline
\end{tabular}

Blank et al.: Radiation Therapy Improves Control in Juvenile Nasopharyngeal Angiofibroma following Surgery

\section{Conflict of Interest Statement}

The authors have no conflicts of interest to declare.

\section{Funding Sources}

The authors have no funding to any research relevant to this case report.

\section{Author Contributions}

Z.B., R.S., and B.N. completed the chart review, wrote the case report, performed the literature review, generated the figures, and formatted the manuscript. M.B. and C.L. oversaw the medical care of the patient and oversaw the drafting of the manuscript. All authors reviewed and approved the final manuscript.

\section{References}

1 Coutinho-Camillo CM, Brentani MM, Nagai MA. Genetic alterations in juvenile nasopharyngeal angiofibromas. Head Neck. 2008;30(3):390-400.

2 Andrews JC, Fisch U, Valavanis A, Aeppli U, Makek MS. The surgical management of extensive nasopharyngeal angiofibromas with the infratemporal fossa approach. Laryngoscope. 1989;99(4):429-37.

3 Radkowski D, McGill T, Healy GB, Ohlms L, Jones DT. Angiofibroma. Changes in staging and treatment. Arch Otolaryngol Head Neck Surg. 1996;122(2):122-9.

4 Yi Z, Fang Z, Lin G, Lin C, Xiao W, Li Z, et al. Nasopharyngeal angiofibroma: a concise classification system and appropriate treatment options. Am J Otolaryngol. 2013;34(2):133-41.

5 Szymanska A, Szymanski M, Czekajska-Chehab E, Szczerbo-Trojanowska M. Two types of lateral extension in juvenile nasopharyngeal angiofibroma: diagnostic and therapeutic management. Eur Arch Otorhinolaryngol. 2015;272:159-66.

6 Giavroglou C, Constantinidis J, Triaridis S, Daniilidis J, Dimitriadis A. [Angiographic evaluation and embolization of juvenile nasopharyngeal angiofibroma]. HNO. 2007;55(1):36-41.

7 Lee JT, Chen P, Safa A, Juillard G, Calcaterra TC. The role of radiation in the treatment of advanced juvenile angiofibroma. Laryngoscope. 2002;112(7 Pt 1):1213-20.

8 Amdur RJ, Yeung AR, Fitzgerald BM, Mancuso AA, Werning JW, Mendenhall WM. Radiotherapy for juvenile nasopharyngeal angiofibroma. Pract Radiat Oncol. 2011;1(4):271-8.

9 Lopez F, Triantafyllou A, Snyderman CH, Hunt JL, Suarez C, Lund VJ, et al. Nasal juvenile angiofibroma: current perspectives with emphasis on management. Head Neck. 2017;39:1033-45.

10 Hansen EK, Roach M. Handbook of evidence-based radiation oncology. New York, NY: Springer; 2007.

11 Wiatrak BJ, Koopmann CF, Turrisi AT. Radiation therapy as an alternative to surgery in the management of intracranial juvenile nasopharyngeal angiofibroma. Int J Pediatr Otorhinolaryngol. 1993;28(1):51-61.

12 Economou TS, Abemayor E, Ward PH. Juvenile nasopharyngeal angiofibroma: an update of the UCLA experience, 1960-1985. Laryngoscope. 1988;98(2):170-5.

13 Cummings BJ, Blend R, Fitzpatrick P, Clark R, Harwood A, Keane T, et al. Primary radiation therapy for juvenile nasopharyngeal angiofibroma. Laryngoscope. 1984;94:1599-1605.

14 McGahan RA, Durrance FY, Parke RB Jr., Easley JD, Chou JL. The treatment of advanced juvenile nasopharyngeal angiofibroma. Int J Radiat Oncol Biol Phys. 1989;17(5):1067-72.

15 McAfee WJ, Morris CG, Amdur RJ, Werning JW, Mendenhall WM. Definitive radiotherapy for juvenile nasopharyngeal angiofibroma. Am J Clin Oncol. 2006;29(2):168-70.

16 Fields JN, Halverson KJ, Devineni VR, Simpson JR, Perez CA. Juvenile nasopharyngeal angiofibroma: efficacy of radiation therapy. Radiology. 1990;176(1):263-5. 